\title{
Appendix: Semiparametric Regression in Testicular Germ Cell Data
}

\author{
Anastasia Voulgaraki ${ }^{1 *}$, Benjamin Kedem ${ }^{1 *}$, and Barry I. Graubard ${ }^{2}$ \\ ${ }^{1}$ University of Maryland, College Park, MD 20742 \\ avoulgar@math.umd.edu, bnk@math.umd.edu \\ ${ }^{2}$ National Cancer Institute, Rockville, MD 20852 \\ graubarb@exchange.nih.gov
}

February 2012

This appendix contains supplemental material for the paper "Semiparametric Regression in Testicular Germ Cell Data" by Voulgaraki, Kedem, Graubard (2012). It provides formal statements and indication of proofs of the results described in Sections 3.1 and 3.2.

We begin with a basic fact from Fokianos (2004).

Theorem 1. Assume that the regularity conditions in Fokianos (2004) hold. In addition assume that

(a) $\partial^{2} h(\mathbf{x}, \boldsymbol{\theta}) / \partial \boldsymbol{\theta} \partial \boldsymbol{\theta}^{\prime}$ is continuous in a neighborhood of the true parameter,

(b) there is a function $H(\mathbf{x})$ which is integrable with respect to $G_{m}$ and which bounds $\left\|\partial^{2} h(\mathbf{x}, \boldsymbol{\theta}) / \partial \boldsymbol{\theta} \partial \boldsymbol{\theta}^{\prime}\right\|$.

Then

$$
\sqrt{n}\left(\begin{array}{c}
\hat{\boldsymbol{\theta}}-\boldsymbol{\theta}_{0} \\
\hat{\boldsymbol{\mu}}-\boldsymbol{\zeta}
\end{array}\right) \stackrel{D}{\rightarrow} N(\mathbf{0}, \mathbf{W})
$$

as $n \rightarrow \infty$. The matrix $\mathbf{W}$ is defined in Fokianos (2004).

Next, we note that $\tilde{g}_{l}(\mathbf{x})$ admits a convenient representation.

Lemma 1. Assume $\boldsymbol{\theta}$ and $p_{i j}$ are known for $i=1, \ldots, q, m, j=1, \ldots, n_{i}$. Then

$$
\tilde{g}_{l}(\mathbf{x})=\frac{1}{n h_{n}^{p}} \sum_{i=1}^{m} \sum_{j=1}^{n_{i}} \frac{w_{l}\left(\mathbf{x}_{i j}\right)}{\sum_{k=1}^{m} \zeta_{k} w_{k}\left(\mathbf{x}_{i j}\right)} K\left(\frac{\mathbf{x}-\mathbf{x}_{i j}}{h_{n}}\right)
$$

\footnotetext{
${ }^{*}$ Research supported by NSF DMS-1007647
} 
where $\zeta_{k}=n_{k} / n$.

Lemma 2. Assume that $K(\cdot)$ is a nonnegative bounded symmetric function with $\int K(\mathbf{x}) d \mathbf{x}=1, \int \mathbf{x} K(\mathbf{x}) d \mathbf{x}=0, \int \mathbf{x}^{\prime} \mathbf{x} K(\mathbf{x}) d \mathbf{x}=k_{2}>0, \int K^{2}(\mathbf{x}) d \mathbf{x}<\infty$. Assume that $g_{l}$ is continuous and bounded at $\mathbf{x}$. Then

(a) As $n \rightarrow \infty$ and $h_{n} \rightarrow 0$,

$$
E \tilde{g}_{l}(\mathbf{x})=\frac{1}{h_{n}^{p}} \int K\left(\frac{\mathbf{x}-\mathbf{y}}{h_{n}}\right) g_{l}(\mathbf{y}) d \mathbf{y}=g_{l}(\mathbf{x})+o(1) .
$$

(b) If $g_{l}$ is twice continuously differentiable in a neighborhood of $\mathbf{x}$, then as $n \rightarrow \infty$ and $h_{n} \rightarrow 0$,

$$
E \tilde{g}_{l}(\mathbf{x})=g_{l}(\mathbf{x})+\frac{1}{2} h_{n}^{2} \int \mathbf{u}^{\prime} \frac{\partial^{2}}{\partial \mathbf{x} \partial \mathbf{x}^{\prime}} g_{l}(\mathbf{x}) \mathbf{u} K(\mathbf{u}) d \mathbf{u}+o\left(h_{n}^{2}\right) .
$$

(c) As $n \rightarrow \infty, h_{n} \rightarrow 0$ and $n h_{n}^{p} \rightarrow \infty$,

$$
\begin{aligned}
\operatorname{Var}\left(\tilde{g}_{l}(\mathbf{x})\right)= & \frac{1}{n\left(h_{n}\right)^{2 p}} \int \frac{w_{l}^{2}(\mathbf{y})}{\sum_{k=1}^{m} \zeta_{k} w_{k}(\mathbf{y})} K^{2}\left(\frac{\mathbf{x}-\mathbf{y}}{h_{n}}\right) g(\mathbf{y}) d \mathbf{y} \\
& -\frac{1}{n} \sum_{i=1}^{m} \zeta_{i}\left[\int \frac{1}{h_{n}^{p}} \frac{w_{l}(\mathbf{y})}{\sum_{k=1}^{m} \zeta_{k} w_{k}(\mathbf{y})} K\left(\frac{\mathbf{x}-\mathbf{y}}{h_{n}}\right) w_{i}(\mathbf{y}) g(\mathbf{y}) d \mathbf{y}\right]^{2} \\
= & \frac{1}{n h_{n}^{p}} \frac{w_{l}(\mathbf{x}) g_{l}(\mathbf{x})}{\sum_{k=1}^{m} \zeta_{k} w_{k}(\mathbf{x})} \int K^{2}(\mathbf{u}) d \mathbf{u}+o\left(\frac{1}{n h_{n}^{p}}\right) \\
= & \frac{1}{n h_{n}^{p}} \sigma^{2}(\mathbf{x})+o\left(\frac{1}{n h_{n}^{p}}\right)
\end{aligned}
$$

with

$$
\sigma^{2}(\mathbf{x})=\frac{w_{l}(\mathbf{x}) g_{l}(\mathbf{x})}{\sum_{k=1}^{m} \zeta_{k} w_{k}(\mathbf{x})} \int K^{2}(\mathbf{u}) d \mathbf{u} .
$$

Proof of Lemma 2. (a) Part (a) follows by continuity.

(b)

$$
\begin{array}{rll}
E \tilde{g}_{l}(\mathbf{x}) & \stackrel{\operatorname{part}(\text { a) }}{=} & \int K(\mathbf{u}) g_{l}\left(\mathbf{x}-h_{n} \mathbf{u}\right) d \mathbf{u} \\
& \stackrel{\text { 2nd } \stackrel{\text { Taylor exp. }}{=}}{ } & \int K(\mathbf{u})\left[g_{l}(\mathbf{x})-h_{n} \mathbf{u}^{\prime} \frac{\partial g_{l}(\mathbf{x})}{\partial \mathbf{x}}+\frac{h_{n}^{2}}{2} \mathbf{u}^{\prime} \frac{\partial^{2}}{\partial \mathbf{x} \partial \mathbf{x}^{\prime}} g_{l}(\mathbf{x}) \mathbf{u}+o\left(h_{n}^{2}\right)\right] d \mathbf{u} \\
= & g_{l}(\mathbf{x})+\frac{h_{n}^{2}}{2} \int \mathbf{u}^{\prime} \frac{\partial^{2}}{\partial \mathbf{x} \partial \mathbf{x}^{\prime}} g_{l}(\mathbf{x}) \mathbf{u} K(\mathbf{u}) d \mathbf{u}+o\left(h_{n}^{2}\right) .
\end{array}
$$

(c)

$$
\operatorname{Var}\left(\tilde{g}_{l}(\mathbf{x})\right)=\operatorname{Var}\left[\frac{1}{n h_{n}^{p}} \sum_{i=1}^{m} \sum_{j=1}^{n_{i}} \frac{w_{l}\left(\mathbf{x}_{i j}\right)}{\sum_{k=1}^{m} \zeta_{k} w_{k}\left(\mathbf{x}_{i j}\right)} K\left(\frac{\mathbf{x}-\mathbf{x}_{i j}}{h_{n}}\right)\right]
$$




$$
\begin{aligned}
= & \frac{1}{n^{2}\left(h_{n}\right)^{2 p}} \sum_{i=1}^{m} n_{i}\left\{E_{i}\left[\frac{w_{l}^{2}\left(\mathbf{x}_{i 1}\right)}{\left(\sum_{k=1}^{m} \zeta_{k} w_{k}\left(\mathbf{x}_{i 1}\right)\right)^{2}} K^{2}\left(\frac{\mathbf{x}-\mathbf{x}_{i 1}}{h_{n}}\right)\right]\right. \\
& \left.-E_{i}^{2}\left[\frac{w_{l}\left(\mathbf{x}_{i 1}\right)}{\sum_{k=1}^{m} \zeta_{k} w_{k}\left(\mathbf{x}_{i 1}\right)} K\left(\frac{\mathbf{x}-\mathbf{x}_{i 1}}{h_{n}}\right)\right]\right\} \\
= & \frac{1}{n h_{n}^{p}}\left\{\frac{1}{h_{n}^{p}} \int \frac{w_{l}^{2}(\mathbf{y})}{\sum_{k=1}^{m} \zeta_{k} w_{k}(\mathbf{y})} K^{2}\left(\frac{\mathbf{x}-\mathbf{y}}{h_{n}}\right) g(\mathbf{y}) d \mathbf{y}\right. \\
& \left.-\frac{1}{h_{n}^{p}} \sum_{i=1}^{m} \zeta_{i}\left[\int \frac{w_{l}(\mathbf{y})}{\sum_{k=1}^{m} \zeta_{k} w_{k}(\mathbf{y})} K\left(\frac{\mathbf{x}-\mathbf{y}}{h_{n}}\right) w_{i}(\mathbf{y}) g(\mathbf{y}) d \mathbf{y}\right]^{2}\right\} \\
= & \frac{1}{n h_{n}^{p}}\left[\frac{w_{l}^{2}(\mathbf{x}) g(\mathbf{x})}{\sum_{k=1}^{m} \zeta_{k} w_{k}(\mathbf{x})} \int K^{2}(\mathbf{u}) d \mathbf{u}+o(1)\right. \\
& \left.-\sum_{i=1}^{m} h_{n}^{p} \zeta_{i}\left(\frac{w_{l}(\mathbf{x}) g_{i}(\mathbf{x})}{\sum_{k=1}^{m} \zeta_{k} w_{k}(\mathbf{x})}\right)^{2}+o\left(h_{n}^{p}\right)\right] \\
= & \frac{1}{n h_{n}^{p}} \frac{w_{l}(\mathbf{x}) g_{l}(\mathbf{x})}{\sum_{k=1}^{m} \zeta_{k} w_{k}(\mathbf{x})} \int K^{2}(\mathbf{u}) d \mathbf{u}+o\left(\frac{1}{n h_{n}^{p}}\right)
\end{aligned}
$$

by Dominated Convergence Theorem as $h_{n} \rightarrow 0$.

Lemma 3. Assume that $K(\cdot)$ is a nonnegative bounded symmetric function with $\int K(\mathbf{x}) d \mathbf{x}=1, \int \mathbf{x}^{\prime} \mathbf{x} K(\mathbf{x}) d \mathbf{x}=k_{2}>0$. Assume that $g_{l}$ is continuous at $\mathbf{x}$ and bounded, and that the regularity conditions in Fokianos (2004) hold. If the quantity

$$
\mathbf{I}_{r}(\boldsymbol{\theta}, \mu)=\frac{\partial}{\partial \boldsymbol{\theta}_{r}} \frac{w\left(\mathbf{x}_{i j}, \boldsymbol{\theta}_{l}\right)}{1+\sum_{k=1}^{q} \mu_{k}\left[w\left(\mathbf{x}_{i j}, \boldsymbol{\theta}_{k}\right)-1\right]}
$$

is bounded, then $\hat{g}_{l}(\mathbf{x})=\tilde{g}_{l}(\mathbf{x})+O_{p}\left(n^{-1 / 2}\right)$ as $n \rightarrow \infty$ and $h_{n} \rightarrow 0$.

We note that $\mathbf{I}_{r}(\boldsymbol{\theta}, \mu)$ is bounded in the exponential case.

Proof of Lemma 3. We have that

$$
\begin{aligned}
& \hat{g}_{l}(\mathbf{x})-\tilde{g}_{l}(\mathbf{x})= \frac{1}{n h_{n}^{p}} \sum_{i=1}^{m} \sum_{j=1}^{n_{i}}\left[\frac{\hat{w}_{l}\left(\mathbf{x}_{i j}\right)}{1+\sum_{k=1}^{q} \hat{\mu}_{k}\left(\hat{w}_{k}\left(\mathbf{x}_{i j}\right)-1\right)}-\frac{w_{l}\left(\mathbf{x}_{i j}\right)}{\sum_{k=1}^{m} \zeta_{k} w_{k}\left(\mathbf{x}_{i j}\right)}\right] \\
& \times K\left(\frac{\mathbf{x}-\mathbf{x}_{i j}}{h_{n}}\right) \\
&= R_{n}\left(\mathbf{x}_{i j}\right) \\
& \text { 1st } \stackrel{\text { Order }}{=} \sum_{\text {Taylor }}^{q} R_{r=1}\left(\hat{\mu}_{r}-\zeta_{r}\right)+\sum_{r=1}^{q} \mathbf{R}_{2 n r}\left(\hat{\boldsymbol{\theta}}_{r}-\boldsymbol{\theta}_{r}\right)
\end{aligned}
$$

where

$\mathbf{I}_{r}\left(\boldsymbol{\theta}^{*}, \mu^{*}\right)$ 


$$
\begin{aligned}
& =\left.\frac{\partial}{\partial \boldsymbol{\theta}_{r}} \frac{w\left(\mathbf{x}_{i j}, \boldsymbol{\theta}_{l}\right)}{1+\sum_{k=1}^{q} \mu_{k}\left[w\left(\mathbf{x}_{i j}, \boldsymbol{\theta}_{k}\right)-1\right]}\right|_{\substack{\mu_{k}=\mu_{k}^{*} \\
\boldsymbol{\theta}_{k}=\boldsymbol{\theta}_{k}^{*}}} \\
& =\frac{\frac{\partial w\left(\mathbf{x}_{i j}, \boldsymbol{\theta}_{r}^{*}\right)}{\partial \boldsymbol{\theta}_{r}}\left[1+\sum_{k=1}^{q} \mu_{k}^{*}\left(w\left(\mathbf{x}_{i j}, \boldsymbol{\theta}_{k}^{*}\right)-1\right)\right] I(r=l)-w\left(\mathbf{x}_{i j}, \boldsymbol{\theta}_{l}^{*}\right) \mu_{r}^{*} \frac{\partial w\left(\mathbf{x}_{i j}, \boldsymbol{\theta}_{r}^{*}\right)}{\partial \boldsymbol{\theta}_{r}}}{\left(1+\sum_{k=1}^{q} \mu_{k}^{*}\left[w\left(\mathbf{x}_{i j}, \boldsymbol{\theta}_{k}^{*}\right)-1\right]\right)^{2}}
\end{aligned}
$$

and

$$
\begin{aligned}
R_{1 n r} & =-\frac{1}{n h_{n}^{p}} \sum_{i=1}^{m} \sum_{j=1}^{n_{i}} \frac{w\left(\mathbf{x}_{i j}, \boldsymbol{\theta}_{l}^{*}\right)\left[w\left(\mathbf{x}_{i j}, \boldsymbol{\theta}_{r}^{*}\right)-1\right]}{\left(1+\sum_{k=1}^{q} \mu_{k}^{*}\left[w\left(\mathbf{x}_{i j}, \boldsymbol{\theta}_{k}^{*}\right)-1\right]\right)^{2}} K\left(\frac{\mathbf{x}-\mathbf{x}_{i j}}{h_{n}}\right) \\
\mathbf{R}_{2 n r} & =\frac{1}{n h_{n}^{p}} \sum_{i=1}^{m} \sum_{j=1}^{n_{i}} \mathbf{I}_{r}\left(\boldsymbol{\theta}^{*}, \mu^{*}\right) K\left(\frac{\mathbf{x}-\mathbf{x}_{i j}}{h_{n}}\right)
\end{aligned}
$$

and $\left(\mu_{k}^{*}, \boldsymbol{\theta}_{k}^{*}\right)$ is on the line segment between $\left(\hat{\mu}_{k}, \hat{\boldsymbol{\theta}}_{k}\right)$ and $\left(\zeta_{k}, \boldsymbol{\theta}_{k}\right)$. However, it can be shown that both $R_{1 n r}$ and $\mathbf{R}_{2 n r}$ are of the order $O_{p}(1)$. Then, using Theorem 1, the proof is complete

The following lemma will be used in the proof of Theorem 2 .

Lemma 4. Let $E_{i}(\mathbf{x})$ denote the expected value of $\mathbf{x}$ with respect to sample $i$. Then

$$
\begin{aligned}
E_{i}\left|\frac{w_{l}\left(\mathbf{x}_{i 1}\right) K\left(\frac{\mathbf{x}-\mathbf{x}_{i 1}}{h_{n}}\right)}{\sum_{k=1}^{m} \zeta_{k} w_{k}\left(\mathbf{x}_{i 1}\right)}\right|^{2+\delta} & =O\left(h_{n}^{p}\right), \\
\left|\int \frac{w_{l}(\mathbf{y}) K\left(\frac{\mathbf{x}-\mathbf{y}}{h_{n}}\right)}{\sum_{k=1}^{m} \zeta_{k} w_{k}(\mathbf{y})} w_{i}(\mathbf{y}) d G(\mathbf{y})\right|^{2+\delta} & =O\left(h_{n}^{p(2+\delta)}\right) .
\end{aligned}
$$

Proof of Lemma 4. Define

$$
H_{\alpha \beta \gamma}(\mathbf{x})=\frac{1}{n h_{n}^{p}} \sum_{i=1}^{m} \sum_{j=1}^{n_{i}} \frac{w_{l}^{\alpha}\left(\mathbf{x}_{i j}\right) D\left(\mathbf{x}_{i j}\right)}{\left(\sum_{k=1}^{m} \zeta_{k} w_{k}\left(\mathbf{x}_{i j}\right)\right)^{\beta}} K^{\gamma}\left(\frac{\mathbf{x}-\mathbf{x}_{i j}}{h_{n}}\right)
$$

where $D(\cdot)$ is some measurable function. Then

$$
\begin{aligned}
E\left(H_{\alpha \beta \gamma}(\mathbf{x})\right) & =\frac{1}{n h_{n}^{p}} \sum_{i=1}^{m} n_{i} E\left(\frac{w_{l}^{\alpha}\left(\mathbf{x}_{i j}\right) D\left(\mathbf{x}_{i j}\right)}{\left(\sum_{k=1}^{m} \zeta_{k} w_{k}\left(\mathbf{x}_{i j}\right)\right)^{\beta}} K^{\gamma}\left(\frac{\mathbf{x}-\mathbf{x}_{i j}}{h_{n}}\right)\right) \\
& =\frac{1}{h_{n}^{p}} \int \frac{w_{l}^{\alpha}(\mathbf{y}) D(\mathbf{y})}{\left(\sum_{k=1}^{m} \zeta_{k} w_{k}(\mathbf{y})\right)^{\beta-1}} K^{\gamma}\left(\frac{\mathbf{x}-\mathbf{y}}{h_{n}}\right) d G(\mathbf{y}),
\end{aligned}
$$

assuming the integral is finite. Set $\alpha=\beta-1=\gamma=2+\delta$ and $D(\mathbf{y})=w_{i}(\mathbf{y})$ in (3). Then

$$
E_{i}\left|\frac{w_{l}\left(\mathbf{x}_{i 1}\right) K\left(\frac{\mathbf{x}-\mathbf{x}_{i 1}}{h_{n}}\right)}{\sum_{k=1}^{m} \zeta_{k} w_{k}\left(\mathbf{x}_{i 1}\right)}\right|^{2+\delta}=h_{n}^{p} E\left(H_{2+\delta .3+\delta, 2+\delta}\right)=O\left(h_{n}^{p}\right) .
$$


Also, if we replace $\alpha=\beta-1=\gamma=1$ and $D(\mathbf{y})=w_{i}(\mathbf{y})$ in (3),

$$
\left|\int \frac{w_{l}(\mathbf{y}) K\left(\frac{\mathbf{x}-\mathbf{y}}{h_{n}}\right)}{\sum_{k=1}^{m} \zeta_{k} w_{k}(\mathbf{y})} w_{i}(\mathbf{y}) d G(\mathbf{y})\right|^{2+\delta}=\left|E\left(H_{1,2,1}\right) h_{n}^{p}\right|^{2+\delta}=O\left(h_{n}^{p(2+\delta)}\right) .
$$

Theorem 2. Assume that $K(\cdot)$ is a nonnegative bounded symmetric function with $\int K(\mathbf{x}) d \mathbf{x}=1, \int \mathbf{x}^{\prime} \mathbf{x} K(\mathbf{x}) d \mathbf{x}=k_{2}>0$. Assume that $g_{l}$ is continuous at $\mathbf{x}$. If $\int[K(\mathbf{u})]^{2+\delta} d \mathbf{u}<\infty$ for some $\delta>0$, then

$$
\sqrt{n h_{n}^{p}}\left(\hat{g}_{l}(\mathbf{x})-E \tilde{g}_{l}(\mathbf{x})\right) \stackrel{D}{\rightarrow} N\left(\mathbf{0}, \sigma^{2}(\mathbf{x})\right)
$$

as $n \rightarrow \infty, h_{n} \rightarrow 0$ and $n h_{n}^{p} \rightarrow \infty$ with

$$
\sigma^{2}(\mathbf{x})=\frac{w_{l}(\mathbf{x}) g_{l}(\mathbf{x})}{\sum_{k=1}^{m} \zeta_{k} w_{k}(\mathbf{x})} \int K^{2}(\mathbf{u}) d \mathbf{u}
$$

for any fixed $\mathbf{x}$.

Proof of Theorem 2. It suffices to show that

$$
\sqrt{n h_{n}^{p}}\left(\tilde{g}_{l}(\mathbf{x})-E \tilde{g}_{l}(\mathbf{x})\right) \stackrel{D}{\rightarrow} N\left(\mathbf{0}, \sigma^{2}(\mathbf{x})\right)
$$

since, by Lemma 3 , we have that

$$
\sqrt{n h_{n}^{p}}\left(\hat{g}_{l}(\mathbf{x})-E \tilde{g}_{l}(\mathbf{x})\right)=\sqrt{n h_{n}^{p}}\left(\tilde{g}_{l}(\mathbf{x})-E \tilde{g}_{l}(\mathbf{x})\right)+O_{p}\left(\sqrt{h_{n}^{p}}\right) .
$$

Then

$$
\begin{aligned}
\sqrt{n h_{n}^{p}}\left(\tilde{g}_{l}(\mathbf{x})-E \tilde{g}_{l}(\mathbf{x})\right)= & \sum_{i=1}^{m} \sum_{j=1}^{n_{i}}\left[\frac{1}{\sqrt{n h_{n}^{p}}} \frac{w_{l}\left(\mathbf{x}_{i j}\right)}{\sum_{k=1}^{m} \zeta_{k} w_{k}\left(\mathbf{x}_{i j}\right)} K\left(\frac{\mathbf{x}-\mathbf{x}_{i j}}{h_{n}}\right)\right. \\
& \left.-\frac{1}{\sqrt{n h_{n}^{p}}} \int \frac{w_{l}(\mathbf{y})}{\sum_{k=1}^{m} \zeta_{k} w_{k}(\mathbf{y})} K\left(\frac{\mathbf{x}-\mathbf{y}}{h_{n}}\right) w_{i}(\mathbf{y}) d G(\mathbf{y})\right] \\
= & \sum_{i=1}^{m} U_{n i}(\mathbf{x})
\end{aligned}
$$

where

$U_{n i}(\mathbf{x})=\frac{1}{\sqrt{n h_{n}^{p}}} \sum_{j=1}^{n_{i}}\left[\frac{w_{l}\left(\mathbf{x}_{i j}\right)}{\sum_{k=1}^{m} \zeta_{k} w_{k}\left(\mathbf{x}_{i j}\right)} K\left(\frac{\mathbf{x}-\mathbf{x}_{i j}}{h_{n}}\right)-\int \frac{w_{l}(\mathbf{y})}{\sum_{k=1}^{m} \zeta_{k} w_{k}(\mathbf{y})} K\left(\frac{\mathbf{x}-\mathbf{y}}{h_{n}}\right) w_{i}(\mathbf{y}) d G(\mathbf{y})\right]$.

Notice that for $i=1, \ldots, m$ :

$$
E U_{n i}(\mathbf{x})=0
$$

Clearly,

$$
E\left[\sqrt{n h_{n}^{p}}\left(\tilde{g}_{l}(\mathbf{x})-E \tilde{g}_{l}(\mathbf{x})\right)\right]=0 .
$$

Moreover, from Lemma 2: 
- $\operatorname{Var}\left(\sqrt{n h_{n}^{p}}\left(\tilde{g}_{l}(\mathbf{x})-E \tilde{g}_{l}(\mathbf{x})\right)\right)=n h_{n}^{p} \operatorname{Var}\left(\tilde{g}_{l}(\mathbf{x})\right)=\sigma^{2}(\mathbf{x})+o(1)$

- $\operatorname{Var}\left(\sqrt{n h_{n}^{p}}\left(\tilde{g}_{l}(\mathbf{x})-E \tilde{g}_{l}(\mathbf{x})\right)\right)=\sum_{i=1}^{m} \operatorname{Var}\left(U_{n i}(\mathbf{x})\right)=s_{n}^{2}(\mathbf{x})$

where $\mathbf{x}$ is fixed. So $s_{n}^{2}(\mathbf{x})=\sigma^{2}(\mathbf{x})+o(1)$. Observe that:

$$
\begin{aligned}
\sqrt{n h_{n}^{p}}\left(\tilde{g}_{l}(\mathbf{x})-E \tilde{g}_{l}(\mathbf{x})\right)= & \sum_{i=1}^{m} U_{n i}(\mathbf{x}) \\
= & \sum_{j=1}^{n_{1}} \frac{1}{\sqrt{n h_{n}^{p}}}\left(\frac{w_{l}\left(\mathbf{x}_{1 j}\right) K\left(\frac{\mathbf{x}-\mathbf{x}_{1 j}}{h_{n}}\right)}{\sum_{k=1}^{m} \zeta_{k} w_{k}\left(\mathbf{x}_{1 j}\right)}-\int \frac{w_{l}(\mathbf{y}) K\left(\frac{\mathbf{x}-\mathbf{y}}{h_{n}}\right)}{\sum_{k=1}^{m} \zeta_{k} w_{k}(\mathbf{y})} d G_{1}(\mathbf{y})\right) \\
& +\sum_{j=1}^{n_{2}} \frac{1}{\sqrt{n h_{n}^{p}}}\left(\frac{w_{l}\left(\mathbf{x}_{2 j}\right) K\left(\frac{\mathbf{x}-\mathbf{x}_{2 j}}{h_{n}}\right)}{\sum_{k=1}^{m} \zeta_{k} w_{k}\left(\mathbf{x}_{2 j}\right)}-\int \frac{w_{l}(\mathbf{y}) K\left(\frac{\mathbf{x}-\mathbf{y}}{h_{n}}\right)}{\sum_{k=1}^{m} \zeta_{k} w_{k}(\mathbf{y})} d G_{2}(\mathbf{y})\right) \\
& \vdots \\
& +\sum_{j=1}^{n_{m}} \frac{1}{\sqrt{n h_{n}^{p}}}\left(\frac{w_{l}\left(\mathbf{x}_{m j}\right) K\left(\frac{\mathbf{x}-\mathbf{x}_{m j}}{h_{n}}\right)}{\sum_{k=1}^{m} \zeta_{k} w_{k}\left(\mathbf{x}_{m j}\right)}-\int \frac{w_{l}(\mathbf{y}) K\left(\frac{\mathbf{x}-\mathbf{y}}{h_{n}}\right)}{\sum_{k=1}^{m} \zeta_{k} w_{k}(\mathbf{y})} d G_{m}(\mathbf{y})\right) .
\end{aligned}
$$

We will show that Lyapunov's Condition (Billingsley 1995, p. 362) holds using the $c_{r}$-inequality:

$$
E|x+y|^{r} \leq c_{r}\left[E|x|^{r}+E|y|^{r}\right], \text { where } c_{r}= \begin{cases}1, & \text { if } 0<r \leq 1 \\ 2^{r-1}, & \text { if } r>1\end{cases}
$$

We have already showed that $E\left(U_{n i}\right), \operatorname{Var}\left(U_{n i}\right)$ are finite for $i=1,2, \ldots, m$.

For some $\delta>0$, the Lyapunov condition becomes:

$$
\begin{aligned}
& \frac{1}{s_{n}^{2+\delta}}\left[\sum_{j=1}^{n_{1}} E\left|\frac{1}{\sqrt{n h_{n}^{p}}}\left(\frac{w_{l}\left(\mathbf{x}_{1 j}\right) K\left(\frac{\mathbf{x}-\mathbf{x}_{1 j}}{h_{n}}\right)}{\sum_{k=1}^{m} \zeta_{k} w_{k}\left(\mathbf{x}_{1 j}\right)}-\int \frac{w_{l}(\mathbf{y}) K\left(\frac{\mathbf{x}-\mathbf{y}}{h_{n}}\right)}{\sum_{k=1}^{m} \zeta_{k} w_{k}(\mathbf{y})} w_{1}(\mathbf{y}) d G(\mathbf{y})\right)\right|^{2+\delta}\right. \\
& \quad+\quad \sum_{j=1}^{n_{2}} E\left|\frac{1}{\sqrt{n h_{n}^{p}}}\left(\frac{w_{l}\left(\mathbf{x}_{2 j}\right) K\left(\frac{\mathbf{x}-\mathbf{x}_{2 j}}{h_{n}}\right)}{\sum_{k=1}^{m} \zeta_{k} w_{k}\left(\mathbf{x}_{2 j}\right)}-\int \frac{w_{l}(\mathbf{y}) K\left(\frac{\mathbf{x}-\mathbf{y}}{h_{n}}\right)}{\sum_{k=1}^{m} \zeta_{k} w_{k}(\mathbf{y})} w_{2}(\mathbf{y}) d G(\mathbf{y})\right)\right|^{2+\delta} \\
& \quad \vdots \\
& \left.\quad \sum_{j=1}^{n_{m}} E\left|\frac{1}{\sqrt{n h_{n}^{p}}}\left(\frac{w_{l}\left(\mathbf{x}_{m j}\right) K\left(\frac{\mathbf{x}-\mathbf{x}_{m j}}{h_{n}}\right)}{\sum_{k=1}^{m} \zeta_{k} w_{k}\left(\mathbf{x}_{m j}\right)}-\int \frac{w_{l}(\mathbf{y}) K\left(\frac{\mathbf{x}-\mathbf{y}}{h_{n}}\right)}{\sum_{k=1}^{m} \zeta_{k} w_{k}(\mathbf{y})} w_{m}(\mathbf{y}) d G(\mathbf{y})\right)\right|^{2+\delta}\right] \\
& \quad \leq \quad \frac{1}{s_{n}^{2+\delta}} \frac{1}{n^{1+\frac{\delta}{2}} h_{n}^{p\left(1+\frac{\delta}{2}\right)}}\left[n_{1} 2^{\delta+1} E_{1}\left|\frac{w_{l}\left(\mathbf{x}_{11}\right) K\left(\frac{\mathbf{x}-\mathbf{x}_{11}}{h_{n}}\right)}{\sum_{k=1}^{m} \zeta_{k} w_{k}\left(\mathbf{x}_{11}\right)}\right|^{2+\delta}\right. \\
& +\quad n_{1} 2^{\delta+1}\left|\int \frac{w_{l}(\mathbf{y}) K\left(\frac{\mathbf{x}-\mathbf{y}}{h_{n}}\right)}{\sum_{k=1}^{m} \zeta_{k} w_{k}(\mathbf{y})} w_{1}(\mathbf{y}) d G(\mathbf{y})\right|^{2+\delta}
\end{aligned}
$$




$$
\begin{array}{ll}
+ & n_{2} 2^{\delta+1} E_{2}\left|\frac{w_{l}\left(\mathbf{x}_{21}\right) K\left(\frac{\mathbf{x}-\mathbf{x}_{21}}{h_{n}}\right)}{\sum_{k=1}^{m} \zeta_{k} w_{k}\left(\mathbf{x}_{21}\right)}\right|^{2+\delta}+n_{2} 2^{\delta+1}\left|\int \frac{w_{l}(\mathbf{y}) K\left(\frac{\mathbf{x}-\mathbf{y}}{h_{n}}\right)}{\sum_{k=1}^{m} \zeta_{k} w_{k}(\mathbf{y})} w_{2}(\mathbf{y}) d G(\mathbf{y})\right|^{2+\delta} \\
+ & n_{m} 2^{\delta+1} E_{m}\left|\frac{w_{l}\left(\mathbf{x}_{m 1}\right) K\left(\frac{\mathbf{x}-\mathbf{x}_{m 1}}{h_{n}}\right)}{\sum_{k=1}^{m} \zeta_{k} w_{k}\left(\mathbf{x}_{m 1}\right)}\right|^{2+\delta} \\
& \left.+n_{m} 2^{\delta+1}\left|\int \frac{w_{l}(\mathbf{y}) K\left(\frac{\mathbf{x}-\mathbf{y}}{h_{n}}\right)}{\sum_{k=1}^{m} \zeta_{k} w_{k}(\mathbf{y})} w_{m}(\mathbf{y}) d G(\mathbf{y})\right|^{2+\delta}\right] \\
\stackrel{\text { Lemma } 4}{=} & \frac{1}{s_{n}^{2+\delta}} \frac{1}{n^{1+\delta / 2} h_{n}^{p(1+\delta / 2)}}\left[n_{1} 2^{\delta+1} O\left(h_{n}^{p}\right)+n_{1} 2^{\delta+1} O\left(h_{n}^{p(2+\delta)}\right)+\ldots\right. \\
= & \left.+n_{m} 2^{\delta+1} O\left(h_{n}^{p}\right)+n_{m} 2^{\delta+1} O\left(h_{n}^{p(2+\delta)}\right)\right] \\
=\quad O\left(\left(n h_{n}^{p}\right)^{-\delta / 2}\right)
\end{array}
$$

where $E_{i}$ is the expected value with respect to the $i$ sample.

Therefore, as $h_{n} \rightarrow 0, n \rightarrow \infty$ and $n h_{n}^{p} \rightarrow \infty$, by the Lyapunov condition:

$$
\frac{\sqrt{n h_{n}^{p}}\left(\tilde{g}_{l}(\mathbf{x})-E \tilde{g}_{l}(\mathbf{x})\right)}{s_{n}(\mathbf{x})} \stackrel{D}{\rightarrow} N(\mathbf{0}, 1) .
$$

By Slutsky's theorem:

$$
\frac{\sqrt{n h_{n}^{p}}\left(\tilde{g}_{l}(\mathbf{x})-E \tilde{g}_{l}(\mathbf{x})\right)}{\sigma(\mathbf{x})}=\frac{\sqrt{n h_{n}^{p}}\left(\tilde{g}_{l}(\mathbf{x})-E \tilde{g}_{l}(\mathbf{x})\right)}{s_{n}(\mathbf{x})} \frac{\sqrt{\sigma^{2}(\mathbf{x})+o(1)}}{\sigma(\mathbf{x})} \stackrel{D}{\rightarrow} N(\mathbf{0}, 1) .
$$

Corollary 1. Assume that $K(\cdot)$ is a nonnegative bounded symmetric function with $\int K(\mathbf{x}) d \mathbf{x}=1, \int \mathbf{x}^{\prime} \mathbf{x} K(\mathbf{x}) d \mathbf{x}=k_{2}>0$. Assume that $g_{l}$ is continuous at $\mathbf{x}$ and twice differentiable in a neighborhood of $\mathbf{x}$. If, as $n \rightarrow \infty, h_{n}=O\left(n^{-\frac{1}{4+p}}\right)$, then

$$
\sqrt{n h_{n}^{p}}\left(\hat{g}_{l}(\mathbf{x})-g_{l}(\mathbf{x})-\frac{1}{2} h_{n}^{2} \int \mathbf{u}^{\prime} \frac{\partial^{2} g_{l}\left(\mathbf{x}^{*}\right)}{\partial \mathbf{x} \partial \mathbf{x}^{\prime}} \mathbf{u} K(\mathbf{u}) d \mathbf{u}\right) \stackrel{D}{\rightarrow} N\left(\mathbf{0}, \sigma^{2}(\mathbf{x})\right)
$$

as $n \rightarrow \infty$

Proof of Corollary 1. Corollary 1 follows immediately from Theorem 1 and Lemma 2.

Theorem 3. Assume that $K(\cdot)$ is a nonnegative bounded symmetric function with $\int K(\mathbf{x}) d \mathbf{x}=1, \int \mathbf{x}^{\prime} \mathbf{x} K(\mathbf{x}) d \mathbf{x}=k_{2}>0$ and $\int K^{2}(\mathbf{x}) d \mathbf{x}<\infty$. If $g_{l}$ is twice continuously differentiable at $\mathbf{x}$ and the regularity conditions in Fokianos (2004) hold, then 
(a) as $n \rightarrow \infty, h_{n} \rightarrow 0$ and $n h_{n}^{p} \rightarrow \infty$

$$
\begin{aligned}
\operatorname{MISE}\left(\hat{g}_{l}\right)= & \frac{1}{n h_{n}^{p}} \int \frac{w_{l}(\mathbf{x}) g_{l}(\mathbf{x})}{\sum_{k=1}^{m} \zeta_{k} w_{k}(\mathbf{x})} d \mathbf{x} \int K^{2}(\mathbf{u}) d \mathbf{u} \\
& +\frac{h_{n}^{4}}{4} \int\left(\int \mathbf{u}^{\prime} \frac{\partial^{2} g_{l}(\mathbf{x})}{\partial \mathbf{x} \partial \mathbf{x}^{\prime}} \mathbf{u} K(\mathbf{u}) d \mathbf{u}\right)^{2} d \mathbf{x}+o\left(\frac{1}{n h_{n}^{p}}\right)+o\left(h_{n}^{4}\right),
\end{aligned}
$$

(b) by minimizing the sum of the two leading terms in (a) with respect to $h_{n}$, the asymptotically optimal bandwidth is:

$$
h_{n}^{*}=\left(\frac{(p / n) \int w_{l}(\mathbf{x}) g_{l}(\mathbf{x}) /\left[\sum_{k=1}^{m} \zeta_{k} w_{k}(\mathbf{x})\right] d \mathbf{x} \int K^{2}(\mathbf{u}) d \mathbf{u}}{\int\left(\int \mathbf{u}^{\prime}\left(\partial^{2} g_{l}(\mathbf{x}) / \partial \mathbf{x} \partial \mathbf{x}^{\prime}\right) \mathbf{u} K(\mathbf{u}) d \mathbf{u}\right)^{2} d \mathbf{x}}\right)^{\frac{1}{4+p}} .
$$

The mean integrated square error of $\hat{g}_{l}$ with optimal bandwidth $h_{n}^{*}$ is:

$$
\begin{gathered}
\operatorname{MISE}^{*}\left(\hat{g}_{l}\right)=n^{-\frac{4}{4+p}}\left(p^{-\frac{p}{4+p}}+\frac{1}{4} p^{\frac{4}{4+p}}\right)\left(\int \frac{w_{l}(\mathbf{x}) g_{l}(\mathbf{x})}{\sum_{k=1}^{m} \zeta_{k} w_{k}(\mathbf{x})} d \mathbf{x} \int K^{2}(\mathbf{u}) d \mathbf{u}\right)^{\frac{4}{4+p}} \\
\cdot\left(\int\left(\int \mathbf{u}^{\prime} \frac{\partial^{2} g_{l}(\mathbf{x})}{\partial \mathbf{x} \partial \mathbf{x}^{\prime}} \mathbf{u} K(\mathbf{u}) d \mathbf{u}\right)^{2} d \mathbf{x}\right)^{\frac{p}{4+p}}+o\left(n^{-\frac{4}{4+p}}\right) .
\end{gathered}
$$

Proof of Theorem 3. (a)

$$
\begin{aligned}
\operatorname{MISE}\left(\hat{g}_{l}\right)= & E \int\left|\hat{g}_{l}(\mathbf{x})-g_{l}(\mathbf{x})\right|^{2} d \mathbf{x} \\
= & \int\left[E\left(\hat{g}_{l}(\mathbf{x})-\tilde{g}_{l}(\mathbf{x})\right)^{2}+E\left(\tilde{g}_{l}(\mathbf{x})-g_{l}(\mathbf{x})\right)^{2}\right. \\
& \left.+2 E\left(\hat{g}_{l}(\mathbf{x})-\tilde{g}_{l}(\mathbf{x})\right)\left(\tilde{g}_{l}(\mathbf{x})-g_{l}(\mathbf{x})\right)\right] d \mathbf{x}
\end{aligned}
$$

From Lemmas 2 and 3 and Schwartz inequality:

$$
\begin{aligned}
E\left(\hat{g}_{l}(\mathbf{x})-\tilde{g}_{l}(\mathbf{x})\right)^{2}= & O\left(n^{-1}\right) \\
E\left(\tilde{g}_{l}(\mathbf{x})-g_{l}(\mathbf{x})\right)^{2}= & \frac{1}{n h_{n}^{p}} \sigma^{2}(\mathbf{x})+\frac{h_{n}^{4}}{4}\left(\int \mathbf{u}^{\prime} \frac{\partial^{2} g_{l}(\mathbf{x})}{\partial \mathbf{x} \partial \mathbf{x}^{\prime}} \mathbf{u} K(\mathbf{u}) d \mathbf{u}\right)^{2} \\
& +o\left(\frac{1}{n h_{n}^{p}}\right)+o\left(h_{n}^{4}\right) \\
E\left(\hat{g}_{l}(\mathbf{x})-\tilde{g}_{l}(\mathbf{x})\right)\left(\tilde{g}_{l}(\mathbf{x})-g_{l}(\mathbf{x})\right)= & O\left(\left(n^{2} h_{n}^{p}\right)^{-1 / 2}\right)
\end{aligned}
$$

Therefore

$$
\operatorname{MISE}\left(\hat{g}_{l}\right)=\int\left[\frac{1}{n h_{n}^{p}} \sigma^{2}(\mathbf{x})+\frac{h_{n}^{4}}{4}\left(\int \mathbf{u}^{\prime} \frac{\partial^{2} g_{l}(\mathbf{x})}{\partial \mathbf{x} \partial \mathbf{x}^{\prime}} \mathbf{u} K(\mathbf{u}) d \mathbf{u}\right)^{2}\right] d \mathbf{x}+o\left(\frac{1}{n h_{n}^{p}}\right)+o\left(h_{n}^{4}\right)
$$




$$
\begin{aligned}
= & \frac{1}{n h_{n}^{p}} \int \frac{w_{l}(\mathbf{x}) g_{l}(\mathbf{x})}{\sum_{k=1}^{m} \zeta_{k} w_{k}(\mathbf{x})} d \mathbf{x} \int K^{2}(\mathbf{u}) d \mathbf{u} \\
& +\frac{h_{n}^{4}}{4} \int\left(\int \mathbf{u}^{\prime} \frac{\partial^{2} g_{l}(\mathbf{x})}{\partial \mathbf{x} \partial \mathbf{x}^{\prime}} \mathbf{u} K(\mathbf{u}) d \mathbf{u}\right)^{2} d \mathbf{x}+o\left(\frac{1}{n h_{n}^{p}}\right)+o\left(h_{n}^{4}\right)
\end{aligned}
$$

(b) Differentiate $\operatorname{MISE}\left(\hat{g}_{l}\right)$ with respect to $h_{n}$ and set equal to 0 . We may ignore the terms $o\left(\left(n h_{n}^{p}\right)^{-1}\right), o\left(h_{n}^{4}\right)$.

Theorem 4. If $\hat{f}(\mathbf{x})=\frac{1}{n_{l} h_{n}^{p}} \sum_{i=1}^{n_{l}} K\left(\frac{\mathbf{x}-\mathbf{x}_{i}}{h_{n}}\right)$ is the classical single-sample multivariate kernel density estimator of $g_{l}$, and AMISE is the asymptotic mean integrated square error, then As $n \rightarrow \infty, h_{n} \rightarrow 0$ and $n h_{n}^{p} \rightarrow \infty$,

(a)

$$
\operatorname{AMISE}\left(\hat{g}_{l}\right) \leq \operatorname{AMISE}(\hat{f})
$$

(b) Using optimal bandwidths, the proposed semiparametric density estimator $\hat{g}_{l}(\mathbf{x})$ is more efficient than $\hat{f}(\mathbf{x})$, i.e for every $l$

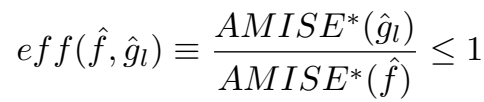

where $A M I S E^{*}$ is the optimal $A M I S E$.

Proof of Theorem 4. (a) According to Cacoullos (1966), if $\hat{f}(\mathbf{x})=\frac{1}{n_{l} h_{n}^{p}} \sum_{i=1}^{n_{l}} K\left(\frac{\mathbf{x}-\mathbf{x}_{i}}{h_{n}}\right)$ is the classic multivariate kernel density estimator of $g_{l}$, then, as $n \rightarrow \infty$, $h_{n} \rightarrow 0$ and $n h_{n}^{p} \rightarrow \infty$ :

$$
\operatorname{AMISE}(\hat{f})=\frac{1}{4} h_{n}^{4} \int\left(\int \mathbf{u}^{\prime} \frac{\partial^{2} g_{l}(\mathbf{x})}{\partial \mathbf{x} \partial \mathbf{x}^{\prime}} \mathbf{u} K(\mathbf{u}) d \mathbf{u}\right)^{2} d \mathbf{x}+\frac{1}{n_{l} h_{n}^{p}} \int K^{2}(\mathbf{x}) d \mathbf{x}
$$

In Theorem 3 we proved that:

$$
\begin{aligned}
\operatorname{AMISE}\left(\hat{g}_{l}\right)=\frac{1}{4} h_{n}^{4} \int\left(\int \mathbf{u}^{\prime} \frac{\partial^{2} g_{l}(\mathbf{x})}{\partial \mathbf{x} \partial \mathbf{x}^{\prime}} \mathbf{u} K(\mathbf{u}) d \mathbf{u}\right)^{2} d \mathbf{x} \\
\quad+\frac{1}{n_{l} h_{n}^{p}} \int \frac{\zeta_{l} w_{l}(\mathbf{x}) g_{l}(\mathbf{x})}{\sum_{k=1}^{m} \zeta_{k} w_{k}(\mathbf{x})} d \mathbf{x} \int K^{2}(\mathbf{x}) d \mathbf{x}
\end{aligned}
$$

Since for every $l, \int \frac{\zeta_{l} w_{l}(\mathbf{x}) g_{l}(\mathbf{x})}{\sum_{k=1}^{m} \zeta_{k} w_{k}(\mathbf{x})} d \mathbf{x} \leq 1$, it follows that

$$
A M I S E\left(\hat{g}_{l}\right) \leq \operatorname{AMISE}(\hat{f}) .
$$


(b) $\operatorname{AMISE}(\hat{f})$ is optimized for $h_{n}=\left[\frac{p}{n_{l}} \frac{\int K^{2}(\mathbf{x}) d \mathbf{x}}{\int\left(\int \mathbf{u}^{\prime} \frac{\partial^{2} g_{l}(\mathbf{x})}{\partial \mathbf{x} \partial \mathbf{x}^{\prime}} \mathbf{u} K(\mathbf{u}) d \mathbf{u}\right)^{2} d \mathbf{x}}\right]^{\frac{1}{p+4}}$. Then, the optimal $A M I S E^{*}(\hat{f})$ is:

$$
\begin{aligned}
\operatorname{AMISE}^{*}(\hat{f})= & {\left[\left(p^{p} n_{l}^{4}\right)^{-\frac{1}{p+4}}+\frac{1}{4}\left(p n_{l}^{-1}\right)^{\frac{4}{p+4}}\right]\left[\int\left(\int \mathbf{u}^{\prime} \frac{\partial^{2} g_{l}(\mathbf{x})}{\partial \mathbf{x} \partial \mathbf{x}^{\prime}} \mathbf{u} K(\mathbf{u}) d \mathbf{u}\right)^{2} d \mathbf{x}\right]^{\frac{p}{p+4}} } \\
& \cdot\left(\int K^{2}(\mathbf{x}) d \mathbf{x}\right)^{\frac{4}{p+4}} .
\end{aligned}
$$

Therefore the asymptotic relative efficiency of $\hat{f}$ with respect to $\hat{g}_{l}$ is given by:

$$
\begin{aligned}
& \text { eff }\left(\hat{f}, \hat{g}_{l}\right) \equiv \frac{A M I S E^{*}\left(\hat{g}_{l}\right)}{A M I S E^{*}(\hat{f})} \\
& =\left[\int \frac{\zeta_{l} w_{l}(\mathbf{x}) g_{l}(\mathbf{x})}{\sum_{k=1}^{m} \zeta_{k} w_{k}(\mathbf{x})} d \mathbf{x}\right]^{\frac{4}{4+p}} \leq 1 \text { for every } l .
\end{aligned}
$$

Thus, unless only the $l$ th sample is available, the proposed semiparametric density estimator is more efficient than the traditional kernel density estimator.

Theorem 5. Assume that the data are bounded. Then:

(a) As $n \rightarrow \infty, h \rightarrow 0$ and $n h^{p} \rightarrow \infty$,

$$
\int|\hat{\mathrm{E}}(y \mid \mathbf{x})-\mathrm{E}(y \mid \mathbf{x})| g(\mathbf{x}) d \mathbf{x} \rightarrow 0
$$

in the mean square sense.

(b) If, in addition $0<A<g(\mathbf{x})$, then

$$
\int|\hat{\mathrm{E}}(y \mid \mathbf{x})-\mathrm{E}(y \mid \mathbf{x})| d \mathbf{x} \rightarrow 0
$$

in the mean square sense.

Proof of Theorem 5. (a) Let $\mathbf{x}$ be a vector of size $k=p-1$ of bounded covariates, and $y$ a bounded response. We have:

$$
\hat{\mathrm{E}}(y \mid \mathbf{x})-\mathrm{E}(y \mid \mathbf{x})=\frac{\frac{1}{n} \sum_{i=1}^{n} y_{i} \hat{g}\left(\mathbf{x}, y_{i}\right)}{\frac{1}{n} \sum_{i=1}^{n} \hat{g}\left(\mathbf{x}, y_{i}\right)}-\frac{\int y g(\mathbf{x}, y) d y}{g(\mathbf{x})}
$$

Or for sufficiently large $n$

$$
\hat{\mathrm{E}}(y \mid \mathbf{x})-\mathrm{E}(y \mid \mathbf{x})=\frac{\int y \hat{g}(\mathbf{x}, y) d y}{\hat{g}(\mathbf{x})}-\frac{\int y g(\mathbf{x}, y) d y}{g(\mathbf{x})} \sim \frac{\int y[\hat{g}(\mathbf{x}, y)-g(\mathbf{x}, y)] d y}{g(\mathbf{x})}
$$


Thus by Cauchy-Schwarz,

$$
\begin{aligned}
{\left[\int|\hat{\mathrm{E}}(y \mid \mathbf{x})-\mathrm{E}(y \mid \mathbf{x})| g(\mathbf{x}) d \mathbf{x}\right]^{2} } & \sim\left[\int\left|\int y[\hat{g}(\mathbf{x}, y)-g(\mathbf{x}, y)] d y\right| d \mathbf{x}\right]^{2} \\
& \leq C \iint|[\hat{g}(\mathbf{x}, y)-g(\mathbf{x}, y)]|^{2} d y d \mathbf{x}
\end{aligned}
$$

Therefore $E\left[\int|\hat{\mathrm{E}}(y \mid \mathbf{x})-\mathrm{E}(y \mid \mathbf{x})| g(\mathbf{x}) d \mathbf{x}\right]^{2} \leq C \cdot M I S E\left(\hat{g}_{l}\right)$. But, by Theorem $3, \operatorname{MISE}\left(\hat{g}_{l}\right)$ converges to 0 as $n \rightarrow \infty, h \rightarrow 0$ and $n h^{p} \rightarrow \infty$, so:

$$
\int|\hat{\mathrm{E}}(y \mid \mathbf{x})-\mathrm{E}(y \mid \mathbf{x})| g(\mathbf{x}) d \mathbf{x} \rightarrow 0
$$

in mean square.

(b) It follows directly from (a) since $g(\mathbf{x})$ is uniformly bounded away from 0 .

\section{References}

[1] Billingsley, P. (1995), Probability and Measure, Wiley-Interscience, New York.

[2] Cacoullos, T.(1966), "Estimation of a multivariate density," Annals of the Institute of Statistical Mathematics (Tokyo), 18(2), 179-189.

[3] Fokianos, K. (2004). Merging information for semiparametric density estimation. Journal of the Royal Statistical Society, Series B, 66, 941-958. 